\title{
Trombocitopenia Aguda Pós-Angioplastia Coronária Primária
}

\author{
Luiz Fernando Ybarra ${ }^{1}$, Antonio Carlos Carvalho ${ }^{1}$
}

\section{RESUMO}

A intervenção coronária percutânea com stent associada ao uso de aspirina, clopidogrel, heparina e inibidores da glicoproteína Ilb/IIla diminuiu a incidência de desfechos desfavoráveis no contexto da síndrome coronária aguda. Contudo, tal associação pode dificultar o manejo do paciente que evolui com plaquetopenia, uma complicação rara do uso do abciximab e da heparina, mas de grande importância clínica. Descrevemos o caso de um paciente com infarto agudo do miocárdio com supradesnivelamento do segmento ST, que evoluiu com plaquetopenia após o início do tratamento. Avaliamos a literatura sobre a diferença da plaquetopenia secundária ao uso do abciximab e da heparina, a fisiopatologia dessa complicação e o manejo dessa condição paradoxal, em que a antiagregação é imperativa em decorrência do uso do stent, e em que pode haver sangramentos significativos ou trombose do stent pela interrupção dos medicamentos.

DESCRITORES: Síndrome coronariana aguda. Anticorpos monoclonais. Trombocitopenia.

\begin{abstract}
Acute Thrombocytopenia Following Primary Coronary Angioplasty

The use of stents in percutaneous coronary interventions, associated with aspirin, clopidogrel, heparin and glycoprotein $\mathrm{Ilb} / \mathrm{IIla}$ inhibitors has reduced the incidence of unfavorable outcomes in acute coronary syndromes. However, this association may hinder the management of patients who develop thrombocytopenia, a rare but clinically important complication of the use of abciximab and heparin. We describe a case of a patient with acute ST-segment elevation myocardial infarction who developed thrombocytopenia after the initial treatment. We evaluated literature data on the difference of thrombocytopenia secondary to the use of abciximab and heparin, the pathophysiology of this complication and the management of this paradoxical condition where antiplatelet therapy is imperative due to the use of stent, but where there may be significant bleeding or stent thrombosis due to drug discontinuation.
\end{abstract}

KEY-WORDS: Acute coronary syndrome. Antibodies, monoclonal. Thrombocytopenia.

infarto, tendo o paciente recebido ou não terapia fibrinolítica. Os antitrombínicos nesse cenário também previnem a trombose venosa profunda, a embolia pulmonar, a formação de trombo ventricular e a embolização cerebral. Todavia, deve-se atentar para seus possíveis efeitos adversos.

Tanto os antagonistas da glicoproteína Ilb/Illa quanto os antitrombínicos podem causar graves complicações. O risco de sangramento e de trombocitopenia deve sempre ser considerado ao se utilizar essas medicações, levando-se em conta as características do paciente e suas comorbidades.

Aproveitamos a descrição de um caso para discutir tanto a plaquetopenia quanto o consequente risco de sangramento causado pelo uso dessas drogas no contexto da síndrome coronária aguda, revendo e reavaliando a literatura do ponto de vista fisiopatológico, clínico, diagnóstico e terapêutico.
1 Universidade Federal de São Paulo - São Paulo, SP, Brasil. Correspondência: Luiz Fernando Ybarra. Rua Capote Valente, 671 ap. 702 - Pinheiros - São Paulo, SP, Brasil - CEP 05409-002 E-mail: Ifybarra@gmail.com

Recebido em: 18/12/2009 • Aceito em: 19/2/2010 


\section{RELATO DE CASO}

Paciente masculino, com 50 anos de idade, admitido com queixa de dor torácica de forte intensidade há 20 minutos, ao repouso, em aperto, com irradiação para os membros superiores, associada a náusea e sudorese. Apresentava antecedente de tabagismo (60 anos-maço) e hipertensão arterial, em uso de enalapril e hidroclorotiazida. O exame físico revelava pressão arterial de $190 \times 130 \mathrm{mmHg}$, sem outras alterações. Foi realizado eletrocardiograma, que demonstrou supradesnivelamento do segmento ST de V1 a V5.

Foi, então, realizado diagnóstico de infarto agudo do miocárdio com supradesnivelamento do segmento ST, administrando-se $300 \mathrm{mg}$ de aspirina, $300 \mathrm{mg}$ de clopidogrel e isordil $5 \mathrm{mg}$ sublingual, e o paciente evoluiu com hipotensão (pressão arterial 80/60 mmHg).

O paciente foi rapidamente encaminhado ao laboratório de hemodinâmica, onde foi realizada angiografia coronária que revelou artéria descendente anterior ocluída no óstio e demais artérias sem estenoses. Foi feita angioplastia da artéria descendente anterior com tempo porta-balão de 68 minutos, com pré-dilatação (balão Maverick 3,0 x $20 \mathrm{~mm}$ ) e stent (Liberté 4,0 x $16 \mathrm{~mm}$ ), sendo administrados 5.000 UI de heparina não-fracionada e abciximab, nas doses convencionais de 20 mg em 10 minutos e manutenção de 10 mcg/min por 12 horas, durante e após o procedimento. A coronariografia de controle demonstrou fluxo TIMI 3 e após o cateterismo o paciente foi encaminhado à unidade de tratamento intensivo (UTI) apresentando tremores e hipertermia.

Durante a evolução na UTI o paciente apresentou plaquetopenia progressiva, que chegou a seu nadir no terceiro dia de internação (Tabela 1). Foi realizado esfregaço de sangue periférico que apresentou contagem de plaqueta de $7 \times 10^{9} /$, com presença de macroplaquetas e poucos grumos plaquetários (Figura 1). Não foram observadas alterações nas séries eritrocítica e granulocítica. Foram suspensos aspirina, clopidogrel e heparina, pelo alto risco de sangramento, embora clinicamente não tenha havido registro de hemorragia, equimoses ou hematomas importantes. Contudo, já no dia seguinte, com a ascensão da contagem plaquetária, tais medicações foram reintroduzidas.

Foram realizados rastreamentos para púrpura trombocitopênica idiopática e para sangramentos menores, em decorrência de queda de 2,2 pontos da hemoglobina e de 4,7 pontos do hematócrito, ambos com resultado negativo. Ultrassonografia abdominal, sorologias para HIV, hepatite B e hepatite C e provas reumatológicas foram feitas, também todas com resultados negativos.

\section{DISCUSSÃO}

A plaquetopenia aguda (contagem de plaquetas $\left.<100 \times 10^{9} / \mathrm{l}\right)$ costuma ter diferentes etiologias. Em pacientes hospitalizados, a plaquetopenia geralmente é parte de um distúrbio multissistêmico, como coagulação intravascular disseminada, ou é secundária a uma complicação do tratamento, como a induzida por droga (heparina, sulfonamidas, ouro, abciximab). O clopidogrel não é descrito como uma droga causadora de trombocitopenia. ${ }^{1}$ Fora do ambiente hospitalar, a apresentação comumente se deve a pseudotrombocitopenia, indução por drogas, púrpura trombocitopênica idiopática ou gestação. Outras causas são doença hepática, síndrome mielodisplásica, síndromes congênitas e infecção pelo HIV.

A trombocitopenia induzida pela heparina é o tipo mais comum de trombocitopenia induzida por drogas e é uma das causas mais comuns de trombocitopenia em pacientes hospitalizados. Apesar disso, é uma complicação pouco frequente, ocorrendo em apenas $2 \%$ a $3 \%$ dos pacientes. ${ }^{2}$ É definida como queda transitória > 50\% na contagem plaquetária que se inicia 5 a 14 dias após o uso de heparina, com normalização dentro de dias ou semanas e desaparecimento dos anticorpos patogênicos da trombocitopenia induzida pela heparina dentro de semanas a poucos meses. ${ }^{3}$

Dois tipos distintos de plaquetopenia são associados à terapia com heparina. ${ }^{4} \mathrm{~A}$ forma mais comum, que pode ocorrer em até $15 \%$ dos pacientes recebendo doses terapêuticas, é um efeito colateral benigno e autolimitado. Esse tipo é dose-dependente, não imunomediado, raramente causa complicações clínicas ou reduções graves das plaquetas, e normalmente não requer interrupção da heparina. Por outro lado, a forma imune da trombocitopenia induzida pela heparina pode, paradoxalmente, causar graves tromboses arteriais, assim como venosas. O mecanismo nesse caso é a interação do anticorpo (normalmente $\lg G$ ) na heparina com o fator plaquetário 4 (FP4) na superfície plaquetária. ${ }^{4}$ Esse complexo pode ativar a coagulação por uma série de mecanismos, incluindo ativação das plaquetas via receptor pla-

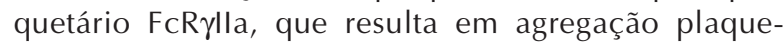
tária, e ativação de células endoteliais e monócitos, resultando em expressão de fator tecidual, início da coagulação e, finalmente, formação de trombina, que leva à amplificação da coagulação e a mais ativação plaquetária. $^{5}$

Os principais fatores de risco para esse distúrbio são: uso da heparina $>4$ dias, uso de heparina nos últimos 100 dias, heparina não-fracionada (a incidência com heparina de baixo peso molecular é $10 \%$ menor), pacientes cirúrgicos e sexo feminino. ${ }^{3}$ O declínio plaquetário normalmente é moderado, com um nadir típico de 50-60 x 10\% / Mais de 90\% dos pacientes com trombocitopenia induzida pela heparina clínica têm queda da contagem plaquetária maior que $50 \%$ do basal durante o tratamento com 
TABELA 1

Evolução dos exames durante a internação

\begin{tabular}{|c|c|c|c|c|c|c|c|}
\hline & Admissão & $2^{o}$ dia & $3^{o}$ dia & $\begin{array}{c}4^{\circ} \text { dia } \\
(6 h 00)\end{array}$ & $\begin{array}{c}4^{\circ} \mathrm{dia} \\
(14 \mathrm{~h} 40)\end{array}$ & $5^{o}$ dia & $6^{0}$ dia \\
\hline Hemoglobina $(\mathrm{g} / \mathrm{dl})$ & 15,7 & 14,8 & 13,8 & 13,5 & 14 & 14,4 & 14,2 \\
\hline Hematócrito (\%) & 43,7 & 42,2 & 39,3 & 39 & 42,9 & 41,8 & 42 \\
\hline Leucócitos (x 109/l) & 18 & 19,2 & 16,6 & 15,3 & 11,3 & 11,5 & 10,2 \\
\hline Plaquetas (x 109/l) & 237 & 33 & 3 & 24 & 89 & 105 & 178 \\
\hline Troponina (ng/ml) & 48,71 & 59,78 & 26,37 & ND & ND & ND & ND \\
\hline CK-MB (U/I) & 325 & 211 & 64 & 25 & ND & 15 & ND \\
\hline Creatinina (mg/dl) & 2,04 & 1,15 & 0,92 & 0,93 & ND & 0,84 & 0,74 \\
\hline RNI & 1,03 & ND & 1 & 1 & ND & 0,98 & 0,97 \\
\hline TTPa (relação) & 0,80 & ND & 0,94 & 0,89 & ND & 0,86 & 0,80 \\
\hline
\end{tabular}

Notar o nadir da trombocitopenia no terceiro dia e a rápida normalização a partir do período da tarde do quarto dia.

CK-MB = creatina quinase fração $\mathrm{MB}$; ND = informação não-disponível; RNI = razão normalizada internacional; TTPa = tempo de tromboplastina parcial ativada.

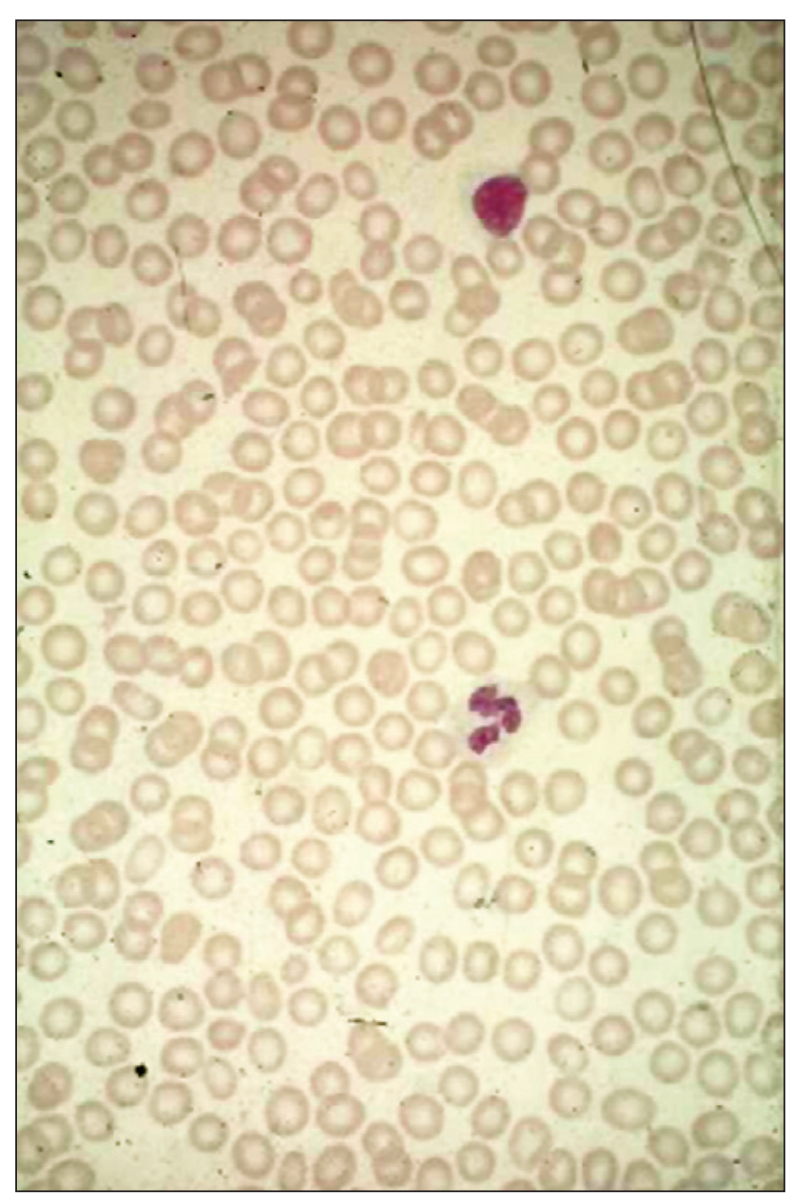

Figura 1 - Lâmina demonstrando plaquetopenia grave (na imagem não são visualizadas plaquetas, denotando contagem plaquetária $<10 \times 10^{9} / \mathrm{l}$ ).

heparina. ${ }^{3}$ Deve-se considerar o diagnóstico quando a trombocitopenia ocorre em um padrão temporal consistente com imunização induzida pela heparina, com queda da contagem plaquetária que começa dentro de 5 a 10 dias (ou trombocitopenia que ocorre em 7 a 14 dias) após o início do uso da terapia com heparina, ou quando ocorre trombose ou qualquer outra sequela de trombocitopenia induzida pela heparina em pacientes tratados com heparina.

Entre $30 \%$ e $50 \%$ dos indivíduos afetados desenvolverão trombose dentro dos 30 primeiros dias do diagnóstico. ${ }^{4} \mathrm{~A}$ forte associação entre trombocitopenia induzida pela heparina e trombose indica que a trombocitopenia induzida pela heparina deva ser suspeitada e que deva ser feita contagem plaquetária (e comparada com os valores dos resultados prévios) em um paciente que desenvolva trombose arterial ou venosa sintomática enquanto recebe heparina profilática ou terapêutica, ou dentro de alguns dias após o uso da heparina. Em aproximadamente 25\% dos pacientes, a trombose associada à trombocitopenia induzida pela heparina precede o desenvolvimento da plaquetopenia em um dia ou em poucos dias. Nesses casos, a administração de doses maiores de heparina para tratar a trombose pode desmascarar a trombocitopenia induzida pela heparina, ao induzir a plaquetopenia mais rapidamente. ${ }^{3}$

Ainda não há um exame laboratorial que faça o diagnóstico de certeza, permacendo a trombocitopenia induzida pela heparina como uma condição de diagnóstico clínico apoiado por exames laboratoriais. ${ }^{4,6}$ Os exames envolvem avaliações funcionais, em que a ativação plaquetária induzida pela heparina in vitro é testada para agregação, liberação de serotonina ou marcadores de ativação plaquetária. Alternativamente, imunoensaios enzimáticos para o complexo anticorpo-heparina-FP4 podem ser utilizados, tendo alta sensibilidade, mas baixa especificidade. 
Quando a trombocitopenia induzida pela heparina é suspeitada, o uso de qualquer tipo de heparina deve ser suspenso imediatamente. $\mathrm{O}$ tratamento da trombocitopenia induzida pela heparina pode ser feito com anticoagulante não-heparínico, como danaparoide, lepirudina, argatroban, fondaparinux ou bivalirudina, ao invés da continuação do uso de heparina não-fracionada ou de heparina de baixo peso molecular ou do início de antagonista da vitamina K. ${ }^{3}$

Em pacientes submetidos a intervenção coronária percutânea, o bloqueio farmacológico da glicoproteína Ilb/Illa surgiu como uma estratégia-chave para melhorar o resultado clínico. No entanto, o aumento da utilização de antagonistas da glicoproteína Ilb/Illa levou ao aumento dos riscos associados, como hemorragia e trombocitopenia. Todos os inibidores da glicoproteína Ilb/IIla (tirofiban, eptifibatide e abciximab) têm sido associados com trombocitopenia aguda, com incidências variando de $0,5 \%$ a 5,6\%, havendo maior incidência com o uso do abciximab que com os demais. ${ }^{1,7,8}$

O abciximab é um fragmento Fab do anticorpo monoclonal quimérico 7E3, que possui especificidade pelo receptor da glicoproteína IIb/IIla situado na superfície celular das plaquetas humanas. Ele se liga a esse receptor e bloqueia a ligação das moléculas de adesão (como o fibrinogênio), inibindo, assim, a agregação plaquetária. A destruição aguda de plaquetas que ocorre após a exposição a um inibidor da glicoproteína Ilb/IIla sugere que fatores não-imunes podem estar em funcionamento. ${ }^{9}$ No entanto, estudos falharam em confirmar essa possibilidade. Relatórios recentes indicam que, na maioria dos casos, a destruição das plaquetas é provocada por anticorpos droga-dependentes. ${ }^{10}$ A plaquetopenia que ocorre após a primeira exposição a um inibidor da glicoproteína Ilb/IIla parece ser explicada pelo fato de os anticorpos estarem presentes naturalmente em alguns indivíduos normais. ${ }^{10}$ Contudo, a maioria dos casos relatados ocorreu numa segunda exposição, especialmente com o abciximab. ${ }^{11}$ A plaquetopenia de início tardio pode ser explicada pela persistência de plaquetas ligadas ao fármaco por várias semanas após o tratamento, tornando-as suscetíveis à destruição pelos anticorpos recémformados. ${ }^{10}$

Vários testes têm sido utilizados para mostrar que existem anticorpos que reconhecem a glicoproteína IIb/IIla ocupada pela droga indutora. O método favorito envolve a detecção de imunoglobulinas, por citometria de fluxo, no soro do paciente e que se ligam às plaquetas normais na presença da droga implicada. Entretanto, os anticorpos não são detectáveis em todos os pacientes, nem mesmo naqueles com história fortemente sugestiva de sensibilidade a drogas. Isso se deve ao fato de não haver ainda definição quanto às condições ideais para a detecção de anticorpos. Um único método pode não ser suficiente para todos os casos, sendo necessários procedimentos especiais para distinguir anticorpos patogênicos capazes de causar trombocitopenia em pacientes que recebem o abciximab daqueles anticorpos aparentemente benignos que apenas reconhecem as plaquetas ligadas ao abciximab. ${ }^{10}$

As variáveis independentes associadas ao desenvolvimento de plaquetopenia após o uso de abciximab são: uso de abciximab nos últimos seis meses, creatinina basal > 0,8 $\mathrm{mg} / \mathrm{dl}$, ataque isquêmico transitório prévio, sexo feminino e história de doença vascular periférica. ${ }^{1}$ Não há dados na literatura demonstrando qualquer diferença no grau de plaquetopenia e desfechos adversos maiores ou menores entre o uso de stent farmacológico e de stent não-farmacológico. O nadir médio da contagem de plaquetas em pacientes tratados com abciximab é de $60 \times 10^{9} / \mathrm{l}$, sendo o mínimo de $3 \times 10^{9} / \mathrm{I}$ e o máximo, de $99 \times 10^{9} /{ }^{1}$

Embora a maioria dos doentes que desenvolvem trombocitopenia após o tratamento com esses medicamentos se recupere sem intercorrências, evoluindo de maneira assintomática ou com sangramentos menores, hemorragias graves e mortais têm sido descritas. Em 30 dias de acompanhamento, um número estatisticamente significante maior de pacientes com plaquetopenia morreu ou precisou de revascularização do vaso-alvo. Esse pior prognóstico demanda acompanhamento mais cuidadoso no primeiro mês após a intervenção. ${ }^{1}$

O manejo desses pacientes depende da gravidade da plaquetopenia e das comorbidades do paciente, assim como da presença e da quantidade de sangramento. Deve-se, inicialmente, prevenir o sangramento, evitando medicações intramusculares, prevenindo quedas e usando laxativos. O desenvolvimento de hemorragia na vigência de plaquetopenia é relacionado à presença de febre, infecção, defeitos de coagulação e lesões anatômicas e ao próprio nível de trombocitopenia. ${ }^{12}$

A diálise e a plasmaférese não se mostraram efetivas em eliminar o abciximab, uma vez que ele se liga de maneira extremamente forte ao receptor da glicoproteína e pouca droga livre está presente, devendo a droga ser suspensa se a plaquetopenia for grave. Caso o paciente apresente contagem plaquetária $<20 \times 10^{9} / \mathrm{l}$ ou $<50 \times 10^{9} / \mathrm{l}$ associada a sangramento, deve-se considerar transfusão de plaquetas e interrupção do abciximab, heparina, aspirina e clopidogrel. Embora ainda não haja consenso sobre quando a reintrodução da terapia antiplaquetária deva ser realizada, considera-se que contagem plaquetária superior a $50 \times 10^{9} / \mathrm{l}$ permite a reintrodução dos antiplaquetários e antitrombínicos. ${ }^{13} \mathrm{Em}$ nosso caso isso ocorreu em pouco mais de 24 horas e junto com sexo masculino, adulto jovem e sem comorbidades contribuiu para a boa evolução do paciente. Caso a ascensão plaquetária seja muito rápida, há risco de gerar um estado pró-trombótico paradoxal, o qual pode coexistir com sangramento ativo. 


\section{CONCLUSÃO}

O manejo de pacientes com stents que evoluem com plaquetopenia é desafiador. A imprevisibilidade da resolução da plaquetopenia induzida pelo abciximab é especialmente difícil. Ainda não há dados suficientes na literatura que comprovem a necessidade de retirada de todos os agentes antiplaquetários e antitrombínicos, muito menos quanto ao nível de plaquetopenia em que se deve fazê-lo. Deve-se levar em consideração o risco de trombose intrastent, a presença de sangramento, seja ele maior ou menor, e a contagem plaquetária. Deve-se, também, monitorar a contagem plaquetária diariamente, pois a identificação precoce de queda grave das plaquetas associada à suspensão dos demais agentes antiplaquetários e antitrombínicos é importante na prevenção de complicações graves. Após a alta hospitalar, especialmente no primeiro mês após a intervenção, a ocorrência de casos de trombose arterial ou venosa agudas, com risco inerente de óbito ou de amputação de membros, exige reconhecimento e tratamento imediatos para reduzir a possibilidade de sequelas graves. Testes de função plaquetária mais acurados podem, no futuro, ser úteis no manejo e no diagnóstico desses pacientes.

Tendo em vista a discussão e a investigação realizadas, assim como a evolução do caso, que apresentou nadir plaquetário no terceiro dia, com contagem de $3 \times 10^{9} / \mathrm{l}$, seguida de rápida normalização, podese inferir como diagnóstico a trombocitopenia secundária ao abciximab. O paciente evoluiu de maneira satisfatória, sem apresentar sangramentos ou sinais de trombose. Contudo, não podemos afirmar que a rápida percepção da plaquetopenia, assim como a interrupção imediata de todas as drogas, abreviou a evolução e evitou complicações.

\section{CONFLITO DE INTERESSES}

Os autores declararam inexistência de conflito de interesses relacionado a este manuscrito.

\section{REFERÊNCIAS}

1. Warkentin TE, Greinacher A, Koster A, Lincoff AM. Treatment and prevention of heparin-induced thrombocytopenia: American College of Chest Physicians Evidence-Based Clinical Practice Guidelines (8 ${ }^{\text {th }}$ Edition). Chest. 2008;133(6 Suppl):340S-80S.

2. Keeling D, Davidson S, Watson $\mathrm{H}$. The management of heparin-induced thrombocytopenia. Br J Haematol. 2006;133(2): 259-69.

3. Arepally GM, Ortel TL. Heparin-induced thrombocytopenia. N Engl J Med. 2006;355(8):809-19.

4. Poncz M. Mechanistic basis of heparin-induced thrombocytopenia. Semin Thorac Cardiovasc Surg. 2005;17(1):73-9.

5. Greinacher A, Warkentin TE. Recognition, treatment, and prevention of heparin-induced thrombocytopenia: review and update. Thromb Res. 2006;118(2):165-76.

6. Merlini PA, Rossi M, Menozzi A, Buratti S, Brennan DM, Moliterno DJ, et al. Thrombocytopenia caused by abciximab or tirofiban and its association with clinical outcome in patients undergoing coronary stenting. Circulation. 2004; 109(18):2203-6.

7. Valgimigli M, Biondi-Zoccai G, Tebaldi M, Van't Hof AWJ, Campo G, Hamm C, et al. Tirofiban as adjunctive therapy for acute coronary syndromes and percutaneous coronary intervention: a meta-analysis of randomized trials. Eur Heart J. 2010;31(1):35-49.

8. Topol EJ, Moliterno DJ, Herrmann HC, Powers ER, Grines $\mathrm{CL}$, Cohen DJ, et al. Comparison of two platelet glycoprotein Ilb/IIla inhibitors, tirofiban and abciximab, for the prevention of ischemic events with percutaneous coronary revascularization. N Engl J Med. 2001;344(25):1888-94.

9. Patrono C, Baigent C, Hirsh J, Roth G. Antiplatelet drugs: American College of Chest Physicians Evidence-Based Clinical Practice Guidelines (8th Edition). Chest. 2008;133(6 Suppl): 199S-233S.

10. Aster RH, Curtis BR, Bougie DW, Dunkley S, Greinacher A, Warkentin TE, et al. Thrombocytopenia associated with the use of GPIIb/IIla. J Thromb Haemost. 2006(3);4:678-9.

11. Dery JP, Braden GA, Lincoff AM, Kereiakes DJ, Browne K, Little $\mathrm{T}$, et al. Final results of the ReoPro readministration registry. Am J Cardiol. 2004;93(8):979-84.

12. Berkowitz SD, Harrington RA, Rund MM, Tcheng JE. Acute profound thrombocytopenia after C7E3 Fab (abciximab) therapy. Circulation. 1997;95(4):809-13.

13. Nowakowski K, Rogers J, Nelson G, Gunalingam B. Abciximabinduced thrombocytopenia: management of bleeding in the setting of recent coronary stents. J Interven Cardiol. 2008; 21(1):100-5. 